\title{
The locus of masking shape-at-a-slant
}

\author{
WILLIAM EPSTEIN and GARY HATFIELD \\ University of Wisconsin, Madison, Wisconsin 53706
}

\begin{abstract}
Twelve subjects provided shape and orientation judgments for a set of projectively equivalent, variously rotated rectangles under three viewing conditions-monoptic, dichoptic, and binocular-with and without the presence of a pattern mask. In the absence of the mask, partial constancy was exhibited under the first two conditions and near perfect constancy under the binocular condition. Orientation was discriminated. Presence of the mask produced projective shape matching and diminished orientation discrimination. It is argued that the site of masking was postchiasmal, and the results are related to previous work with pattern masking of projectively equivalent ellipses.
\end{abstract}

In previous experiments, Epstein and Hatfield (1978) and Epstein, Hatfield, and Muise (1977) reported masking of simple shapes rotated in depth. When followed by a frontoparallel pattern mask at the appropriate interstimulus interval (ISI), objectively different, projectively identical shapes-at-aslant were indistinguishable. The targets all appeared to conform to projective shape. The targets, which were rotated in depth as much as $65^{\circ}$, tended to appear to have a frontoparallel orientation.

We proposed that the mask affected perceived shape by preempting the visual system before the optical correlates of slant-in-depth could be processed. We assumed in our account that the site of the masking effect was postchiasmal. The aim of the experiment described here was to test this assumption. For this purpose, we employed the conventional paradigm. In this paradigm, inferences concerning the locus of an effect are based on a comparison between two conditions: (1) dichoptic viewing, which exposes the target to one eye and the mask to the other, and (2) monoptic viewing, which exposes the target and the mask to the same eye. If masking effects of equal magnitude are observed under dichoptic and monoptic conditions, we may infer that the site of the effect is postchiasmal. The absence or diminution of masking for dichoptic viewing would implicate a retinal locus. A binocular viewing condition which exposed the target and the mask to both eyes was included to serve as a baseline for constancy and masking effects.

It was expected that only partial constancy would be obtained in the two monocular (monoptic, dichoptic) conditions. Epstein and Hatfield (1978) used projectively equivalent ellipses as stimuli and found that, at exposure durations varying from 25 to $75 \mathrm{msec}$, binocular viewing yielded nearly perfect constancy and monocular viewing without a mask

This research was supported by Research Grant MH 26703 from the National Institute of Mental Health. yielded projective shape matching. In unreported pilot experiments it was found that, at exposures of up to $500 \mathrm{msec}$, monocular viewing of ellipses yielded projective shape matching. Projective shape matches persisted for most subjects even when optical texture was introduced to the surfaces of the ellipses in an attempt to provide orientation information in the monocular field. In order to obtain a degree of constancy under the monocular conditions of the present experiment that would allow detection of the potential effects of the mask, it was necessary to introduce targets which provide effective monocular cues for orientation. Pilot work had suggested that the increased perspective information for orientation afforded by rectangular targets was suitable for our purposes.

The experiment conformed to the following general plan. Each subject was tested under all three viewing conditions. The first stage of testing was devoted to determining the minimum exposure duration that would yield high levels of shape discrimination without a mask. Exposure durations were determined for each subject individually; this yielded a Brunswik ratio' above .70 under both binocular and monocular (dichoptic, monoptic) conditions. Only after these durations were established did testing begin.

\section{METHOD}

\section{Subjects}

Seventeen undergraduates participated in the experiment for course credit, money ( $\$ 2.50$ per session), or a combination of the two at their option.

\section{Stimuli}

The standard stimuli were four rectangles cut from $1 / 16$ in. white posterboard and affixed to $21 / 2$-in.-high black stalks. The stimuli were beveled to minimize edge cues. The length of the vertical axis of each standard was $5.3 \mathrm{~cm}$. The horizontal axes varied from 4.0 to $8.0 \mathrm{~cm}$. The four stimuli had horizontal: vertical axis ratios of $.77, .88,1.08$, and 1.57. Each of these shapes was presented at only one orientation, $0^{\circ}$ (frontoparallel),
} 
$30^{\circ}, 45^{\circ}$, and $60^{\circ}$, respectively. All objective stimuli were rotated counterclockwise. The objective shape-objective orientation combinations were such that, for binocular viewing, all shapes projected a horizontal:vertical ratio of .77 (vertical subtense of $4.5^{\circ}$, horizontal subtense of $3.5^{\circ}$ ) onto the frontal plane. While the four rectangles were equivalent when projected orthogonally onto the frontoparallel plane, the projection of the shapes onto the retina was subject to perspective distortions which, for rotated stimuli, yielded a set of trapezoids. The (vertically oriented) bases of the projected trapezids became increasingly disparate in length with increased rotation from the frontal plane. Furthermore, from the vantage point of the right eye (monoptic condition), the rotation of each standard was $2.5^{\circ}$ less than the figure given above and hence the projective shapes of the rotated stimuli were slightly greater than .77 . For the left eye (dichoptic condition), the rotations were $2.5^{\circ}$ greater and the projective shapes were slightly less than .77 .

The masking stimulus consisted of randomly shaped and randomly arranged pieces of white paper, each less than $1 \mathrm{~cm}^{2}$ in size, attached to black matte construction paper.

The comparison stimuli for shape were nine scaled-down drawings, each with a vertical axis of $2.5 \mathrm{~cm}$. The horizontal axes varied to produce shapes with horizontal:vertical ratios ranging from .6 to 2.0. Four shapes corresponding to the horizontal:vertical axis ratios of the stimuli were embedded within the nine comparison shapes. The comparison stimuli were arranged on single sheets of paper in a single column, either in ascending or descending order.

The comparison stimuli for orientation were six circles $(4.5 \mathrm{~cm}$ in diameter), each containing a single line drawn along a diameter. Each drawing represented a top view of the stimulus at one orientation relative to the observer. The represented orientations ranged from $0^{\circ}$ to $75^{\circ}$ counterclockwise in $15^{\circ}$ increments. The stimuli were arranged on a single sheet of paper in either ascending or descending order.

Individual sheets of comparison stimuli were prepared for each trial and placed in a predetermined random order in a looseleaf binder such that only the current trial information was available to the subject. Each comparison stimulus was numbered. The subject verbally reported his response to each trial and the experimenter recorded it.

\section{Apparatus}

The apparatus was a modified two-field tachistoscope controlled by an Automated Data Systems 1248 timer. Each arm of the tachistoscope was $54 \mathrm{~cm}$ long, $23.5 \mathrm{~cm}$ wide, and $25 \mathrm{~cm}$ high. A light-proof extension of the direct-view channel provided access to a calibrated disk into which the standard shapes could be inserted. The axis of rotation for each standard was $68 \mathrm{~cm}$ from the viewer's eyes. The masking stimulus was in an extension of the reflected arm of the tachistoscope at the same optical distance as the standard. The subject viewed the stimuli through a $10 \times 4 \mathrm{~cm}$ window framed by a viewing hood which excluded ambient light and restricted head movement. For the monoptic condition, cardboard screens were placed in such a way that the standard and mask were visible to the right eye only. For the dichoptic condition, the screens were placed so that the standard was visible only to the left eye and the mask was visible only to the right eye. The overall viewing field was restricted to the standard and masking stimulus by occluders appropriately placed in each channel of the tachistoscope. Illumination was provided by a fluorescent light in each arm, located in front of and below the stimuli. The luminance level in each display field was $3.0 \mathrm{~cd} / \mathrm{m}^{2}$.

\section{Exposure Durations}

On all trials calling for a mask, the mask was exposed for 400 msec. When no mask was presented, offset of the standard was followed by an empty dark field. Exposure durations and SOA were determined individually for each subject under each viewing condition in the manner explained below. The mean exposure durations were 90,98 , and $38 \mathrm{msec}$ for the monoptic, dichoptic, and binocular conditions, respectively. The mean SOAs were 1,3 , and $-4 \mathrm{msec}$ for the monoptic, dichoptic, and binocular conditions, respectively. ${ }^{2}$

\section{Procedure}

Each subject served in three sessions of approximately $1 \mathrm{~h}$ on successive days. The instructions emphasized phenomenal (apparent) shape and orientation judgments. The subject was directed to base the response on an immediate impression of shape and orientation. The first session was devoted to determining the appropriate exposure duration and SOA for each subject under each of the three viewing conditions. For each condition, we determined the minimim exposure duration that allowed a high degree of constancy defined as a Brunswik ratio of .70 or higher. In this way, we were assured that the level of shape discrimination in the absence of a mask was comparable under the three conditions. Had we adopted a single exposure duration for all conditions, performance in the absence of masking would have differed, creating difficulties in interpreting any differences among conditions when the mask was present. In addition, we determined an SOA that, when combined with this exposure duration, yielded a mask-induced reduction of the Brunswik ratio by at least .20 from the no-mask level. ${ }^{3}$ Subjects were dismissed if they failed to exhibit constancy in the absence of the mask or if they could not be masked in the binocular condition. Three were dismissed for the first reason, two for the second, leaving 12 subjects.

In the second and third sessions, each subject was tested in the binocular, monoptic, and dichoptic conditions. On each day, each of the three conditions was represented by three blocks of eight trials. The randomly ordered test trials in each block consisted of the four standards presented with and without the mask. There were nine blocks on each day, and these were counterbalanced within and among subjects in order to avoid position effects. Different permutations of the test trials were used for all the sessions. Eight practice trials were presented on each day, followed by the 72 test trials. Shape and slant judgments of the standards were elicited on each trial.

\section{RESULTS}

\section{Shape Matches}

Table 1 shows the group mean horizontal:vertical $(\mathrm{H}: \mathrm{V})$ axis ratios of the matches under the three viewing conditions with and without the mask. In the absence of the mask, the standard shapes were discriminated. For binocular viewing, the matches conformed closely to the shape constancy function. For monoptic and dichoptic viewing, which did not provide access to retinal dispárity, discrimination of shape was not was good. The matches under these conditions represented a compromise between objective shape and projective shape. The Brunswik ratio is a straightforward index of this tendency. For example, the Brunswik ratios averaged for the three rotated stimuli were $.71, .72$, and 1.06 for the monoptic, dichoptic, and binocular conditions. Reduced constancy is the typical result of cue reduction, and the orientation judgments presented in Table 2 show that orientation under the monoptic and dichoptic conditions was judged less accurately under these latter conditions than under the binocular condition.

The effect of the mask was striking. The mask eliminated shape discrimination entirely. For each of 
Table 1

Mean Ratio of Horizontal to Vertical Axes of Shape Matches Under Three Viewing Conditions With and Without Masking

\begin{tabular}{|c|c|c|c|c|c|c|c|}
\hline \multirow{2}{*}{\multicolumn{2}{|c|}{ H/V Ratio of Standard }} & \multicolumn{6}{|c|}{ Viewing Conditions } \\
\hline & & \multicolumn{2}{|c|}{ Monoptic } & \multicolumn{2}{|c|}{ Dichoptic } & \multicolumn{2}{|c|}{ Binocular } \\
\hline Objective & Projective & Mean & SD & Mean & SD & Mean & SD \\
\hline \multicolumn{8}{|c|}{ Without Mask } \\
\hline $\begin{array}{r}.77 \\
.88 \\
1.08 \\
1.57\end{array}$ & $\begin{array}{l}.77 \\
.77 \\
.77 \\
.77\end{array}$ & $\begin{array}{r}.982 \\
.995 \\
1.044 \\
1.143\end{array}$ & $\begin{array}{l}.220 \\
.144 \\
.224 \\
.255\end{array}$ & $\begin{array}{r}.967 \\
.943 \\
1.044 \\
1.152\end{array}$ & $\begin{array}{l}.187 \\
.125 \\
.218 \\
.285\end{array}$ & $\begin{array}{r}.947 \\
.989 \\
1.138 \\
1.410\end{array}$ & $\begin{array}{l}.136 \\
.138 \\
.281 \\
.373\end{array}$ \\
\hline \multicolumn{8}{|c|}{ With Mask } \\
\hline $\begin{array}{r}.77 \\
.88 \\
1.08 \\
1.57\end{array}$ & $\begin{array}{l}.77 \\
.77 \\
.77 \\
.77\end{array}$ & $\begin{array}{l}.958 \\
.950 \\
.970 \\
.989\end{array}$ & $\begin{array}{l}.128 \\
.107 \\
.190 \\
.174\end{array}$ & $\begin{array}{l}.948 \\
.938 \\
.932 \\
.974\end{array}$ & $\begin{array}{l}.196 \\
.186 \\
.191 \\
.225\end{array}$ & $\begin{array}{r}.945 \\
.953 \\
1.007 \\
.991\end{array}$ & $\begin{array}{l}.155 \\
.149 \\
.259 \\
.288\end{array}$ \\
\hline
\end{tabular}

the three conditions, projective shape functions (zero slope) were obtained. The most telling comparison, insofar as the question of the locus of masking is concerned, involves the monoptic vs. the dichoptic condition. In both cases, binocular disparity is excluded, the only difference being that the monoptic condition allows retinal interaction while the dichoptic condition does not. An additional advantage of this comparison is that the levels of performance in the absence of the mask were highly comparable.

An analysis of variance was performed for the data from the monoptic and dichoptic viewing conditions. The main effect of Standard was significant $[F(3,1136)=18.15, p<.001]$, as was the main effect of Masking Condition $[F(1,1136)=43.29$, $p<.001]$. As would be expected from the equivalence of the scores under the two viewing conditions, the main effect of Viewing Condition was not significant, and this factor did not interact with any other factor. The Mask by Standard interaction was significant $[F(3,1136)=8.73, p<.001]$. For the standard in the frontal plane, the objective and projective horizontal:vertical ratios were equal, and thus the masking conditions had little effect. As the disparity between objective and projective shape in- creased, the subjects exhibited partial constancy in the absence of the masks, and thus the difference between their judgments under the mask and nomask conditions increased.

\section{Orientation Matches}

There was an overall tendency to underestimate the degree of slant-in-depth of the rotated shapes. When the mask was absent, underestimation was least marked for binocular viewing and most marked for monoptic viewing. Despite these inaccuracies, the four standard orientations were discriminated under all the viewing conditions when the mask was absent. Introduction of the mask resulted in deterioration of orientation discrimination for all viewing conditions. This may be most readily noted by examining the grand means listed in the right-hand column of Table 2.

\section{DISCUSSION}

Comparison of shape judgments for binocular viewing with the two monocular viewing conditions (monoptic, dichoptic) indicates that disparity was an important source of orientation information in the binocular viewing condition. Yet there was

Table 2

Mean Orientation Matches in Degrees Under Three Viewing Conditions With and Without Masking

\begin{tabular}{|c|c|c|c|c|c|c|c|c|}
\hline \multicolumn{2}{|c|}{ Standard } & \multicolumn{2}{|c|}{ Monoptic } & \multicolumn{2}{|c|}{ Dichoptic } & \multicolumn{2}{|c|}{ Binocular } & \multirow{2}{*}{$\begin{array}{l}\text { Grand } \\
\text { Mean }\end{array}$} \\
\hline H/V Ratio & Orientation & Mean & SD & Mean & SD & Mean & SD & \\
\hline \multicolumn{9}{|c|}{ Without Mask } \\
\hline $\begin{array}{r}.77 \\
.88 \\
1.08 \\
1.57\end{array}$ & $\begin{array}{r}0 \\
30 \\
45 \\
60\end{array}$ & $\begin{array}{r}8.12 \\
14.17 \\
27.08 \\
30.00\end{array}$ & $\begin{array}{l}16.30 \\
12.56 \\
18.44 \\
18.50\end{array}$ & $\begin{array}{r}6.46 \\
24.79 \\
35.83 \\
42.92\end{array}$ & $\begin{array}{l}11.21 \\
19.20 \\
18.42 \\
18.21\end{array}$ & $\begin{array}{r}3.75 \\
25.41 \\
37.08 \\
48.33\end{array}$ & $\begin{array}{r}8.99 \\
13.71 \\
19.17 \\
18.54\end{array}$ & $\begin{array}{r}6.11 \\
21.46 \\
33.33 \\
40.42\end{array}$ \\
\hline $\begin{array}{r}.77 \\
.88 \\
1.08 \\
1.57\end{array}$ & $\begin{array}{r}0 \\
30 \\
45 \\
60\end{array}$ & $\begin{array}{l}12.92 \\
17.71 \\
21.67 \\
24.38\end{array}$ & $\begin{array}{l}17.68 \\
14.31 \\
20.59 \\
19.73\end{array}$ & $\begin{array}{c}\text { With M } \\
21.46 \\
26.88 \\
26.04 \\
29.17\end{array}$ & $\begin{array}{l}20.73 \\
22.23 \\
18.50 \\
19.32\end{array}$ & $\begin{array}{l}12.71 \\
19.38 \\
26.66 \\
29.58\end{array}$ & $\begin{array}{l}16.05 \\
14.56 \\
18.88 \\
19.50\end{array}$ & $\begin{array}{l}15.70 \\
21.00 \\
24.79 \\
27.71\end{array}$ \\
\hline
\end{tabular}


sufficient orientation information in the monocular field for achievement of partial constancy in the monoptic and dichoptic conditions. Since introduction of the mask yielded an equivalent result under these two conditions, the effect of the mask was not dependent upon the interaction of stimulation in a single retina and hence was postchiasmal. Backward masking of forms in the dichoptic mode has been found for frontoparallel targets and masks by other investigators (Turvey, 1973, pp. 9-10, reviews these results). The present results are the first to show the effect for forms rotated in depth.

Epstein and Hatfield (1978) and Epstein, Hatfield, and Muise (1977) discussed the effects of masking shape-at-a-slant in terms of the shape-slant invariance hypothesis and an algorithm processing model (Epstein \& Hatfield, 1978, Figure 4). The processing stages of this model are (1-3) separate registration, encoding, and transmission to algorithm processor of orientation and projective shape information, and (4) combination of the two kinds of information by application of a shape-slant algorithm. Since projective shape matches were consistently obtained in the masking conditions of the present and previous experiments, the mask did not operate by interfering with the registration, encoding, or transmission of projective shape information. The effective orientation information in the previous experiments was binocular disparity and so the site of masking obviously was postchiasmal. On the basis of the finding that the mask led to a tendency toward frontoparallelness in orientation judgments, it was suggested that the mask operated by preempting orientation encoding.

It is obvious that the orientation information in the monocular conditions of the present experiment was not binocular disparity, and comparison with previous work as discussed in the introduction suggests that perspective was the crucial source of information. The finding that masking was postchiasmal when perspective was the crucial source of orientation information is consistent with location of masking at the state of orientation encoding, though location of the effect at some later stage cannot be ruled out. Further experimentation is required to differentiate among the stages implied in the above-mentioned process model.

\section{REFERENCES}

Epstein, W., \& Hatfield, G. Functional equivalence of masking and cue reduction in perception of shape at a slant. Perception \& Psychophysics, 1978, 23, 137-144.

Epstein, W., Hatrield, G., \& Muise, G. Perceived shape at a slant as a function of processing time and processing load. Journal of Experimental Psychology: Human Perception and Performance, 1977, 3, 473-483.

TURVEY, M. T. On peripheral and central processes in vision: Inferences from an information-processing analysis of masking with patterned stimuli. Psychological Review, 1973, 80, 1-52.

\section{NOTES}

1. The Brunswik ratio is $(a-p) /(o-p)$, in which a is the shape of the match, $p$ is the projective shape, and $o$ is the objective shape, all expressed as the ratio of the horizontal to the vertical axis.

2. A negative SOA signifies that the onset of the mask preceded the onset of the target. In this respect, our current procedure differs from the procedure in our earlier experiments in which the mask always followed onset of the target. Rectangular targets seem harder to mask under the conditions of our experiment than elliptical targets.

3. It should be noted that subjects were not preselected for dichoptic masking, i.e., no subjects were dismissed for failing to exhibit dichoptic masking. Of course, once a subject exhibited dichoptic masking in the preliminary trials, it was not surprising to observe comparable effects in the experimental trials. Nevertheless, the preliminary trials devoted to masking were only few in number and could not serve to provide reliable estimates of the magnitude of the masking effect.

(Received for publication April 28, 1978; revision accepted July 17,1978 .) 